\title{
Primary Renal Neuroblastoma Mimicking Wilms Tumor
}

\author{
Kanwaljeet Kaur ${ }^{1}$ Jagdish Prasad Meena ${ }^{1}$ Prasanth Sri ${ }^{1} \quad$ Aditya Kumar Gupta ${ }^{1}$ Manisha Jana ${ }^{2}$ \\ Venkateswaran K. Iyer ${ }^{3}$ Rakesh Kumar ${ }^{4}$ Rachna Seth ${ }^{1}$ \\ ${ }^{1}$ Division of Pediatric Oncology, Department of Pediatrics, All India \\ Institute of Medical Sciences, New Delhi, India \\ ${ }^{2}$ Department of Radiology, All India Institute of Medical Sciences, \\ New Delhi, India \\ ${ }^{3}$ Department of Pathology, All India Institute of Medical Sciences, \\ New Delhi, India \\ ${ }^{4}$ Department of Nuclear Medicine, All India Institute of Medical \\ Sciences, New Delhi, India \\ Ind J Med Paediatr Oncol 2021;42:380-384. \\ Address for correspondence Jagdish Prasad Meena, MD, \\ Department of Pediatrics, Teaching Block, All India Institute of \\ Medical Sciences, New Delhi 110029, India \\ (e-mail: drjpmeena@yahoo.com).
}

\begin{abstract}
Neuroblastoma (NB) is the most common extracranial solid malignancy in children younger than 5 years of age. It is an aggressive malignancy with evidence of secondary metastasis at the time of the initial presentation. NB is rightly known as a great masquerader. Herein, we describe three children who presented with renal masses mimicked as Wilms tumor initially and later diagnosed as NB on biopsy. The response to therapy was not satisfactory in all three children. A higher level of awareness and

Keywords

- renal neuroblastoma

- Wilms tumor

- diagnostic dilemma

- mimicked early recognition is important for diagnosing and managing NB. We should rule out NB when there is a diagnostic dilemma before nephrectomy in these patients. Intrarenal NB should be considered with the combination of renal mass and hypertension with elevated catecholamines. The distinction between these two tumors is important since both malignancies have different therapeutic and prognostic implications.
\end{abstract}

\section{Introduction}

Neuroblastoma (NB) is the most common malignant abdominal neoplasm in infancy and early childhood, and it constitutes 8 to $10 \%$ of childhood cancers. ${ }^{1}$ It is well known for its varied clinical presentations. Around $65 \%$ of primary tumors are found in adrenals, and the remaining originate anywhere in the body along the sympathetic chain. ${ }^{1}$ Renal NB is believed to originate from either sequestration of adrenal medullary tissue in the kidney during fetal development or intrarenal sympathetic ganglion or a spread of aggressive NB infiltrating into the kidney. ${ }^{2} \mathrm{NB}$ is rightly known as a great masquerader of Wilms tumor clinically and radiologically.
NB arising from the kidney or large tumor that completely replaces the kidney may also resemble Wilms tumor and cause diagnostic dilemma and improper management. Both the tumors occur in the early pediatric age group, with Wilms tumor having a little older peak incidence at between 3 to 4 years. Pathologically, they are different diseases, with NB originating from the primordial neural crest and Wilms tumor arising from mesodermal tumors. ${ }^{3}$ Although NB, compared with Wilms tumor, is an aggressive malignancy, it usually presents with metastasis at the time of presentation in around $75 \%$ of cases. Hereby, we discuss three cases who presented with renal masses and posed a diagnostic dilemma and delayed management.
DOI https://doi.org/ $10.1055 / \mathrm{s}-0041-1735438$ ISSN 0971-5851
(C) 2021. Indian Society of Medical and Paediatric Oncology.

This is an open access article published by Thieme under the terms of the Creative Commons Attribution-NonDerivative-NonCommercial-License, permitting copying and reproduction so long as the original work is given appropriate credit. Contents may not be used for commercial purposes, or adapted, remixed, transformed or built upon. (https://creativecommons.org/licenses/by-nc-nd/4.0/).

Thieme Medical and Scientific Publishers Private Ltd. A-12, Second Floor, Sector -2, NOIDA -201301, India 


\section{Clinical Details of Patients}

\section{Case 1}

A 6-year-old girl presented to our hospital with a complaint of left-sided mild abdomen pain and abdomen lump for 1 month. There was a history of low-grade fever and weight loss of $\sim 10 \%$ over 1.5 months. She denied any history of hematuria. At presentation, she had a large, hard, and nontender mass occupying the left half of the abdomen with rounded discrete margins. She did not have hypertension.

Baseline complete blood counts, liver function tests, and kidney function tests were normal. Serum lactate dehydrogenase (LDH) was $1311 \mathrm{U} / \mathrm{L}$. Urine VMA (vanillylmandelic acid)/ creatinine ratio was $1.0 \mathrm{mg} / \mathrm{g}$ of creatinine (not elevated). The serum ferritin was $1375 \mathrm{ng} / \mathrm{ml}$. The bone marrow examination did not reveal any tumor cells. Contrast-enhanced computerized tomography (CECT) scan of the chest and abdomen revealed a large heterogeneous mass of $11.5 \times 9.5 \mathrm{~cm}$ with large areas of central necrosis arising from the left kidney. The mass appeared to displace and insinuate between the abdominal aorta and inferior vena cava (IVC) without any vascular invasion. The chest CT scan showed multiple nodules, $1.07 \mathrm{~cm} \times 0.7 \mathrm{~cm}$ in the right upper lobe and another in the right middle and lower lobe. Overall, the CT findings were suggestive of Wilms tumor with lung metastasis (-Fig. 1). Metaiodobenzylguanidine (MIBG) scan did not show any MIBG avid lesion.

With the initial possibility of Wilms tumor, she was started on chemotherapy as per International Society of Pediatric Oncology (SIOP) protocol (weekly vincristine, actinomycin-D, and doxorubicin) after taking a biopsy from the mass. After two cycles of the above chemotherapy, the NMYC amplification report came out to be amplified. The patient was then switched to the Vincristine, Cisplatin, Etoposide, Cyclophosphamide (OPEC)/Vincristine, Carboplatin, Etoposide, Cyclophosphamide (OJEC) regimen. The biopsy tumor cells were diffusely immunopositive for chromogranin and synaptophysin, while negative for Wilms tumor-1. The final impression was NB.

After four cycles of OPEC/OJEC, the tumor was not amenable to surgery. After 7 months from the presentation to us, she lost to follow-up.

\section{Case 2}

A 3-year-old male child presented to us with fever, abdominal distension, and fast breathing for the last 2 months. He also had abdominal pain. There was a history of blood transfusion in this child. He had hypertension. His abdomen

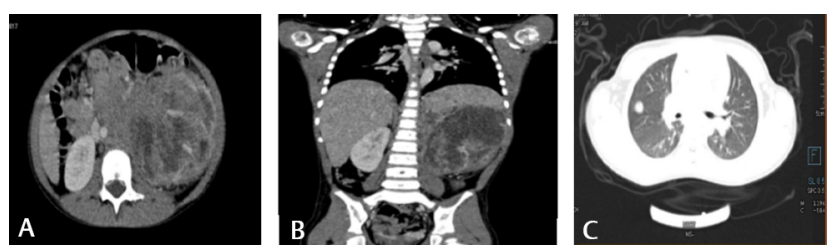

Fig. 1 Contrast-enhanced computed tomography (CECT) abdomen showing left renal mass with necrosis ( $\mathbf{A}$ and $\mathbf{B})$ and right lung metastasis $(\mathbf{C})$. was distended. There was an immobile, nontender mass palpable in the left hypochondrium. Serum ferritin was $3,432 \mathrm{ng} / \mathrm{mL}$, and the urinary VMA was elevated.

The CECT abdomen and chest suggested left renal mass with intrathoracic extension with left pleural effusion and pleural based mass. The possibility of Wilms tumor was considered, and the child was started on chemotherapy for Wilms tumor (SIOP protocol: vincristine and actinomycin-D) in another hospital, and the patient was referred to our hospital. In view of inadequate response to chemotherapy, he was planned for re-evaluation. A biopsy was done, which was suggestive of NB. Cytogenetic analysis showed the amplification of MYCN. The bone marrow aspiration and biopsy did not show the presence of metastases. He was started on the CDCE cycle (cisplatin, cyclophosphamide, doxorubicin, and etoposide). For hypertension, he was started on amlodipine, enalapril, and labetalol. However, there was no response in size of mass or respiratory distress. Subsequently, he was started on OJEC chemotherapy, to which he had a suboptimal response. Parents were counseled regarding poor prognosis in view of high-risk disease and no response to first-line chemotherapy. After a discussion with his parents, he was planned for salvage chemotherapy. He was started on salvage chemotherapy consisting of irinotecan and temozolomide. Even after salvage chemotherapy, there was no response. Finally, we decided on palliative care for him and discharged him on home-based oxygen.

\section{Case 3}

A 5-year-old male child presented with fever, pallor, and progressive abdominal distension for the last 10 days. He had bilateral cervical lymphadenopathy (maximum size $3 \mathrm{~cm}$ ). The abdomen was distended, and he had a hard lump in the right hypochondriac and iliac fossa, not moving with respiration, and it was nontender.

The CECT abdomen showed a large heterogeneously enhancing mass with renal claw sign; the mass was encasing the aorta, IVC, and celiac trunk. A provisional diagnosis of Wilms tumor or NB was considered. Urine VMA was not elevated (9.3 mg). Serum ferritin (761 ng/mL) and LDH (5823 U/L) were elevated. The NMYC was not amplified. Bone marrow examination showed the presence of metastatic infiltration by the tumor. The histopathology report confirmed the diagnosis of NB. He was staged as high-risk (stage-IV) NB. The child was given cytoreductive chemotherapy followed by definitive chemotherapy in the form of an OPEC-OJEC regimen. After the fourth cycle of chemotherapy, he had progressive disease.

\section{Discussion}

The treatment and prognosis for NB and Wilms tumors are very diverse. Primary renal NB carries a worse prognosis and requires intensive chemotherapy. It is important to make the distinction between these two at the earliest. In more than $90 \%$ of cases, NB is MIBG avid, and it is associated with elevated urine VMA. ${ }^{4}$ Compared with Wilms tumor, hypertension is more common, and pulmonary metastasis is uncommon, 
382 Renal Neuroblastoma Mimicking Wilms Tumor Gaur et al.

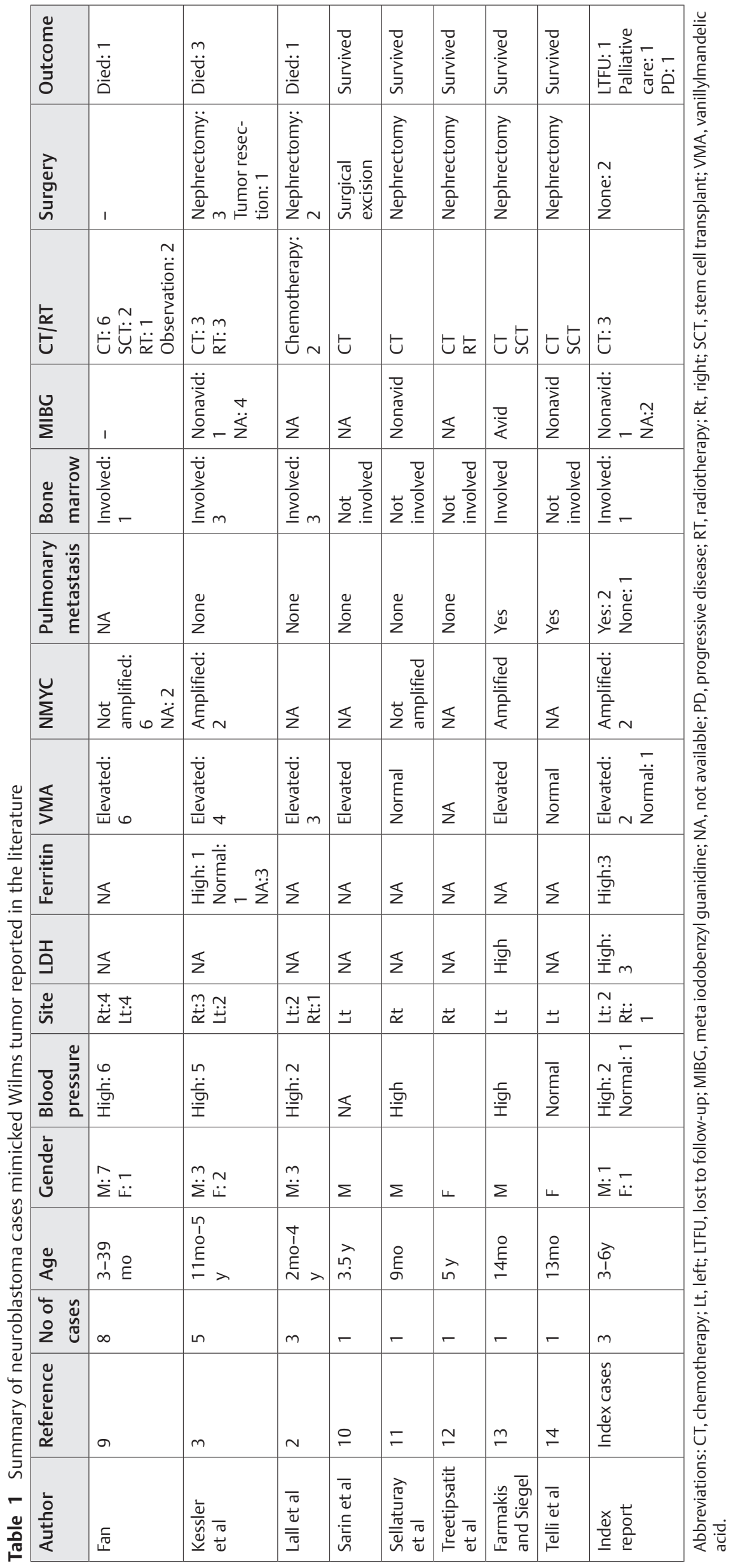

Indian Journal of Medical and Paediatric Oncology Vol. 42 No. 4/2021 C 2021. Indian Society of Medical and Paediatric Oncology. 
and the patient is generally sicker in NB. ${ }^{3}$ However, 5 to $10 \%$ of cases of NB may present with diagnostic ambiguity. ${ }^{2}$ A diagnostic dilemma and inadequate management happen in children with cancer when a relatively stable child presents with renal mass with normal blood pressure and pulmonary metastasis and negative MIBG and normal urine VMA levels. Our first patient presented with renal mass, normal blood pressure, pulmonary metastasis, and normal urine VMA. Case 2 presented with renal mass with high blood pressure, pulmonary metastasis, and elevated VMA. Case 3 presented with normal blood pressure, without pulmonary metastasis, and normal VMA.

Renal NB is of two types. It may result from primary intrarenal NB (IRNB) or renal invasion by NB. The IRNB may result from either sequestered adrenal rests during the fetal life or intrarenal sympathetic ganglia. ${ }^{2}$ Complete renal invasion by NB may also masquerade as Wilms tumor. ${ }^{5}$ Moreover, primary IRNB is usually associated with a poor prognosis as the tumor presents with secondary metastasis at the time presentation of. Our first case responded to chemotherapy (pulmonary metastasis disappeared), but the tumor was not amenable to surgery after four cycles of chemotherapy (tumor was encasing to renal vessels). Case 2 also did not respond to chemotherapy, including salvage therapy, and case 3 progressed after four cycles of chemotherapy.

The primary renal involvement by NB is reported in 5.2 to $25 \%$ of cases. ${ }^{6}$ The renal involvement correlates with large size of the primary tumor, lymph node involvement, undifferentiated histology, and NMYC status. ${ }^{5}$ In a series of 868 NB cases by Shamberger et al, 1 to $2 \%$ cases were presented as IRNB, and they suggested that preoperative chemotherapy may reduce the number of nephrectomies for an initial impression of Wilms tumor. ${ }^{6}$ In the German NB trial of 1,603 NB patients, 29 patients (1.8\%) were those who were initially diagnosed and treated as Wilms tumor. In this study, the postchemotherapy surgery of mass revealed NB. These were mostly advanced-stage disease (stage-III or stage-IV), older age at diagnosis with higher levels of LDH, ferritin, and higher incidence of NMYC amplification than those upfront managed as NB. In this study, the prognosis of patients with NB was reported inferior compared with tumors that have been radiologically classified as Wilms tumor. ${ }^{7}$ All three cases of ours had a high LDH level and high ferritin. Our cases 1 and 2 had NMYC amplification and inadequate response to therapy. However, case 3 was NMYC nonamplified but had progressive disease after four cycles of chemotherapy.

Lung metastasis in NB is very rare, and patients with lung metastasis are sicker, and the prognosis of these patients is worse. Lung metastasis at the initial diagnosis of NB was associated with NMYC amplification, metastasis to central nervous system, elevated LDH levels, and poor outcome. ${ }^{8}$ It was associated with an inferior outcome but not independent of tumor biology. The same parameters were also present in our cases 1 and 2 . The summary of NB cases mimicked Wilms tumor is depicted in - Table 1.

The incidence of hypertension (66-100\%) is more in IRNB compared with extrarenal NB (27\%). ${ }^{3}$ However, this finding is not specific, and it also occurs in $20 \%$ of patients with Wilms tumor. ${ }^{13}$ Testing of urine catecholamines (VMA and HVA) is crucial to further make the diagnosis of NB. ${ }^{9}$ If any patient presents with renal mass and hypertension with high catecholamines, one should strongly suspect NB because these levels are elevated in around $90 \%$ of cases with NB. ${ }^{15}$

Renal NB presents with the high stage at diagnosis as all our cases had advanced stages, and this entity portrays poor outcomes. Primary renal NB carries a poor prognosis, and it requires rigorous adjuvant therapy and close surveillance postoperatively. It is very important to make the distinction between NB and Wilms tumor as treatment and prognosis have differed in these two. ${ }^{9}$ All three cases were initially diagnosed as Wilms tumor and later diagnosed to have NB. All three cases in our series had a poor prognosis.

\section{Conclusion}

It is a diagnostic challenge to distinguish renal NB and Wilms tumor preoperatively. Proper histopathological diagnosis of a renal tumor is important as the management and prognosis of Wilms tumor and NB are different. IRNB should be considered with the combination of renal mass with hypertension and elevated catecholamines. Primary renal NB is associated with unfavorable biology and outcome. Besides, upfront nephrectomy in IRNB may lead to unnecessary loss of the kidney. In doubtful cases of Wilms tumor, the biopsy should always be performed to prevent such errors in judgments.

\section{Funding}

None.

\section{Declaration of Patient Consent}

Legally Authorized Representatives (LARs) have given their consent for publication of this paper. It is available if requested.

\section{Authors' Contribution}

KR, PS, and JPM designed the study and wrote the manuscript. VKI made a histopathologic diagnosis of cases, and MJ provided images of patients. AKG and RS supervised the manuscript writing, and all the authors approved the manuscript.

\section{Conflict of Interest}

None declared.

\section{Acknowledgments}

We would like to express our most incredible gratitude to our patients for supporting us and provide information for the paper's completion. We also would like to thank our colleagues and supervisors for giving support and feedback for this paper.

\section{References}

1 MIBG and Somatostatin Receptor Analogs in Children: Current Concepts on Diagnostic and Therapeutic Use | Journal of Nuclear Medicine [Internet]. [cited 2021 Feb 18]. Available from: https://jnm.snmjournals.org/content/46/1_suppl/55S. Accessed July 18, 2021 
2 Intrarenal neuroblastoma - a diagnostic dilemma: A report of three cases Lall A, Bajpai M, Gupta DK - Indian J Urol [Internet]. [cited 2020 Oct 3]. Available from: https://www.indianjurol. com/article.asp? issn=0970-1591; year $=2001$; volume $=17$; issue $=2 ;$ spage $=170$; epage $=172$; aulast $=$ Lall. Accessed July 18, 2021

3 Kessler OJ, Siegel JF, Brock WA. Intrarenal neuroblastoma masquerading as Wilms' tumor. Urology 1998;51(2):313-316 Available from: http://www.sciencedirect.com/science/article/pii/S0090429597006900 cited 20200ct3 [Internet]

4 Sharp SE, Trout AT, Weiss BD, Gelfand MJ. MIBG in neuroblastoma diagnostic imaging and therapy. Radiographics 2016;36(1):258-278 Available from: https://pubs.rsna.org/ doi/full/10.1148/rg.2016150099 cited 20200ct4 [Internet]

5 Albregts AE, Cohen MD, Galliani CA. Neuroblastoma invading the kidney. J Pediatr Surg 1994;29(7):930-933

6 Shamberger RC, Smith EI, Joshi VV, et al. The risk of nephrectomy during local control in abdominal neuroblastoma. J Pediatr Surg 1998;33(2):161-164

7 Hero B, Graf N, Simon T, Weirich A, Tröger J, Berthold F. Neuroblastoma preoperatively treated as nephroblastoma: does inadequate therapy worsen the prognosis? Klin Padiatr 2002;214(4):157-161

8 Dubois SG, London WB, Zhang Y, et al. Lung metastases in neuroblastoma at initial diagnosis: a report from the International Neuroblastoma Risk Group (INRG) project. Pediatr Blood Cancer 2008;51(5):589-592

9 Fan R. Primary renal neuroblastoma-a clinical pathologic study of 8 cases. Am J Surg Pathol 2012;36(1):94-100
10 Sarin YK, Sinha A, Sengar M. Intrarenal neuroblastoma : a case report. J Indian Assoc Pediatr Surg 2002;7(2):76, Available from https://www.jiaps.com/article.asp?issn=0971-9261;year=2002; volume $=7$;issue $=2$; spage $=76$; epage $=79$; aulast $=$ Sarin;type $=0$ cited 2021 Feb 19 [Internet]

11 Sellaturay SV, Arya M, Banisadr S, Murthi GV, Sebire NJ, Duffy PG. Primary intrarenal neuroblastoma: a rare, aggressive tumour of childhood mimicking Wilms' tumour. J Pediatr Urol 2006;2(5):522-524 Available from: http://www.sciencedirect.com/science/article/pii/S1477513105001865 cited 20200ct5 [Internet]

12 Treetipsatit J, Pradniwat K, Laohapensang M, Rongviriyapanich C, Phuakpet K, Sanpakit K. Primary intrarenal/perirenal neuroblastoma mimicking Wilms' tumor at presentation in a 5-yearold girl: a case report from Siriraj hospital. J Med Assoc Thai 2014;97(9):982-987 Available from: https://linkinghub.elsevier.com/retrieve/pii/S0031302516314313 cited 20200ct5 [Internet]

13 Farmakis SG, Siegel MJ. Intrarenal neuroblastoma with pulmonary metastases mimicking a Wilms tumor. J Pediatr Surg 2014;49(12):1864-1866 Available from: http://www.sciencedirect.com/science/article/pii/S0022346814006940 cited 20200ct5 [Internet]

14 Telli O, Ateş U, Ergun E, et al. Primary intrarenal neuroblastoma in a 13-month-old girl presenting as a Wilms' tumor with pulmonary metastasis. Turk J Pediatr 2016;58(5):532-534

15 Dickson PV, Sims TL, Streck CJ, et al. Avoiding misdiagnosing neuroblastoma as Wilms tumor. J Pediatr Surg 2008;43(6):1159-1163 